\title{
Screening for Carcinoma Cervix
}

\section{Shashi Joshi ${ }^{1 *}$ and Jose Manuel Perez ${ }^{2}$}

${ }^{1}$ Consultant Gynaecologist, J.P. Surgical Hospital, Ambala City, India

${ }^{2}$ Department of Biochemical Studies, City University New York, USA

*Corresponding Author: Shashi Joshi, Consultant Gynaecologist, J.P. Surgical

Hospital, Ambala City, India
Received: January 20, 2021

Published: February 26, 2021

(C) All rights are reserved by Shashi Joshi and Jose Manuel Perez.

\section{Abstract}

Cervical cancer is the second most common cancer in women. It is largely preventable by different screening, diagnostic and therapeutic procedures. It is the only gynaecological cancer that can be prevented through routine screening. The precursor lesions detected take a long time to progress to cancer and can be treated. Hence screening reduces both the incidence and mortality of cervical cancer. Till now cytology-based screening has been the main stay for screening of cervical cancer. Pap smear screening has reduced the incidence of cervical cancer by $80 \%$ and deaths by $70 \%$. In many low resource settings, it is difficult to maintain high quality and high coverage of cytology-based screening programmes. This limitation can be removed by use of FRD as a screening device.

Keywords: Cervical Cancer; FRD; Pap Smear Screening

\section{Abbreviations}

FRD: Folate Receptor Device; TCT: Thinprep Cytology Testing; VIA: Visual Inspection with Acetic Acid; HPV: Human Papilloma Virus; CIN: Cervical Intraepithelial Neoplasia

\section{Introduction}

Screening means testing all women at risk of cervical cancer, the majority of whom will be asymptomatic. Screening aims to detect precancerous changes, which can be treated to avoid progress to cancer. Screening proves to be effective, if there is a well organised system for follow up and treatment and the programme should involve atleast 70 to $80 \%$ of population.

Cervical cancer is the second most common cancer in the females, worldwide. Approximately 530,000 new cases and 275,000 deaths occur each year in the women living in middle and low income group countries [1,2].
Methods available for screening

1. Pap smear

2. Liquid based cytology

3. HPV DNA testing

4. Visual inspection after staining with acetic acid or lugol's iodine

5. FRD.

Recently a novel approach named folate receptor mediated staining solution has been proposed as an effective screening method for CIN and cancer, proven with higher specificity than HPV and TCT $[3,4]$.

\section{Conventional cytology}

Cytological testing is done by Pap Smear. Physician collects the cells from the cervix and smears them on a microscopic slide. A 
fixative is applied and sent to the lab for evaluation [5]. Sensitivity is $50 \%$ and specificity $94 \%$.

A study published in 2007, suggested doing Pap smear, produces cytokine inflammatory response. This may cause immunological clearance of HPV, hence reducing the progress to cervical cancer. A statistically significant decline in HPV positivity rate was correlated with lifetime number of Pap smears received [6].

Thin prep cytology testing

Cells taken from the cervix are placed in a menthol based liquid medium that preserves the cells. The sample is stained and examined microscopically. It has the advantage of being suitable for high risk HPV testing and may reduce unsatisfactory results from 4.1\% to $2.6 \%$ [7].

\section{Limitations of cytology:}

1. Low sensitivity of a single smear.

2. Low reproducibility.

3. High false positive as multiple benign lesions mimic the neoplastic cells. These include atypia of repair, atrophy, radiation changes, effect of IUCD and metaplasia.

\section{HPV testing}

HPV is the causative factor for cervical cancer, in nearly all the cases [8]. Most women will get rid of the infection by 18 months. Those infected with high risk group (i.e. HPV type $16,18,31,45$ ) are likely to have prolonged infection and progress to malignancy [9].

Sensitivity is $88 \%$ to $91 \%$ for CIN 3 or higher and $97 \%$ for CIN2+ and specificity is $73 \%$ to $79 \%$ for CIN $3+$ or higher and $93 \%$ for CIN2+ $[10,11]$.

HPV is time consuming and requires sophisticated lab facilities, compared to other methods. False -ve results reported with HPV testing are less compared to other methods [12].

\section{Visual inspection}

Acetic acid or Lugol's iodine can be used to view precancerous cervical lesions by observing the colour change with the naked eye. Doctors, nurses, trained midwives can effectively perform this test after adequate training. Physicians and mid-level providers can identify between $47 \%$ to $79 \%$ of women at risk of cervical cancer [13]. Like cytology, VIA is also affected by individual interpretation.
VIA can offer significant advantage over Pap smear in low resource setting. There is increased screening coverage and improved follow up care. Need for specialised personnel, infra structure, training and equipment is less. Hence it can be provided in remote, less equipped health care centres. Since there is immediate interpretation of results, the number of women who miss out on treatment, because they can't visit the clinic another time, is reduced.

VIA shows low specificity and high false positive as compared to cytology. Inflammation, condylomata and leukoplakia can give false positive results [14]. VIA has limitations since the endocervical canal can't be visualised, posing a major problem especially in postmenopausal women in whom the endocervical canal has receded.

\section{Folate receptor device}

Folate receptor mediated solution is used for detecting neoplastic changes in cervical epithelium and is mainly composed of folic acid and methylene blue.

Structure and composition the folate receptor-mediated detection (FRD ${ }^{\mathrm{TM}}$ ) staining solution is mainly composed of folic acid, reduced methylene blue, acetic acid, ascorbic acid and dimethyl sulfoxide. It is dispense by GY Highland biotech.

The mechanism of action involves the following steps:

1. Folic acid reduces methylene blue conjugate, binds to folate receptors expressed on cervical epithelial membrane and endocytosis occurs.

2. Acidic microenvironment in the endosomes, causes dissociation of the folic acid and reduces methylene blue from folate receptors.

3. Reduced methylene blue is released into cytoplasm, where oxidation-reduction occurs and reduced methylene blue becomes oxidized.

4. Folate receptors recycle back to cell membrane.

5. Oxidised methylene blue exits the cell and can be detected, providing visual results.

To minimize as much as possible the interpreted variability in identifying colour changes for determining FDR+ve and FDR -ve, the $2^{\text {nd }}$ generation FRD utilises unique shaped epithelial applicator, tapered gradually towards the distal end which is better shaped to fit the ecto and endocervix to ensure satisfactory staining of the 


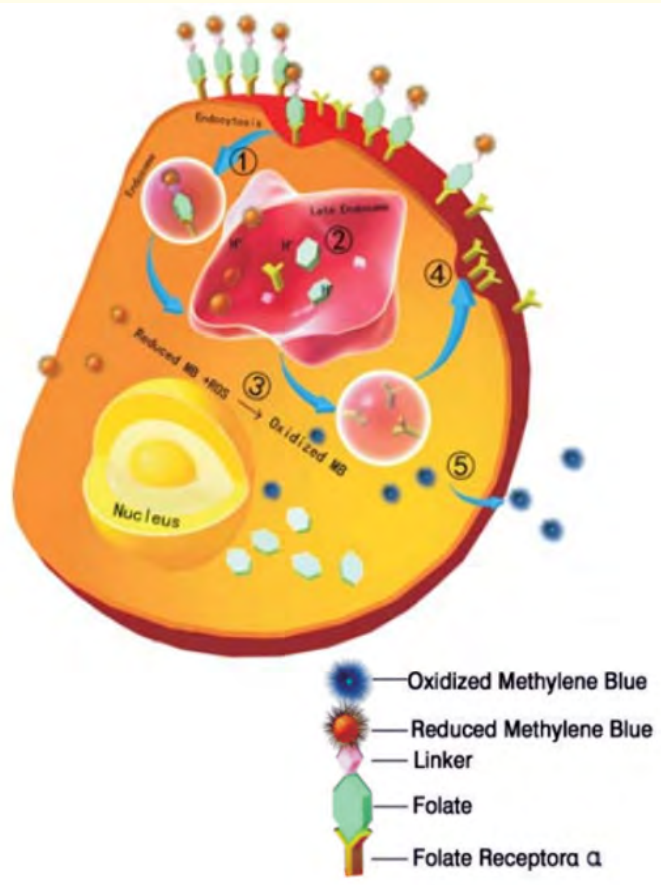

Figure 1

neoplastic epithelia of the whole cervix. Proximal surface of the epithelium staining applicator is labelled so that corresponding location of abnormal cervical lesion can be identified.

Comparing to biopsy, the coincidence rate of FRD in detection of CIN -1, CIN-2, CIN-3, infiltrating carcinoma were $66.7 \%, 75 \%$,

\section{FRD'M Components}
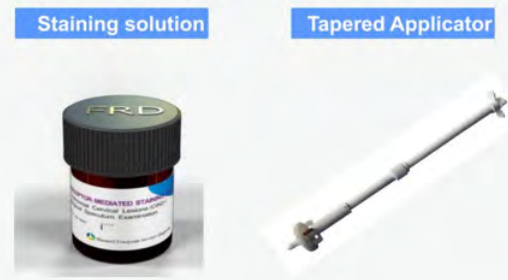

\section{Sampling and staining}

1. Place the patient in the lithotomy position. Expose the cervix with the aid of a speculum and clean any excessive secretion with dry cotton or gauze.

2. Take the Epithelium Staining Applicator, and align the red-marked support bar on the proximal surface of the applicator, to the virtual 12 o'clock position determined on the ectocervix. Insert the applicator into the cervical canal until the polyurethane foam fully covers the ectocervix.
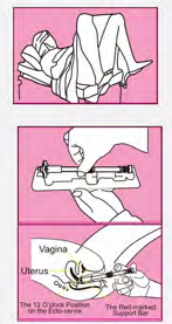

\section{Sampling and staining}

3. Hold the handle and press the applicator on the surface of the cervix for 5 seconds to ensure close contact between the applicator and the ectocervix.

4. Push the plunger and push the applicator against the cervix for 10 seconds. Rotate the plunger 30 degrees to the right and push again for 5 seconds. Then remove the applicator.

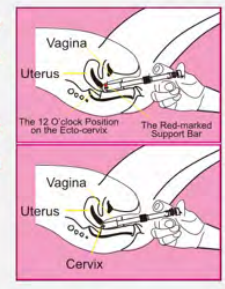

\section{Sampling and staining}

5. Open the brown glass bottle which contains the FRD ${ }^{\mathrm{TM}}$ staining solution. Dip the Epithelium Staining Applicator (which contains the sample) into the FRD ${ }^{\mathrm{TM}}$ staining solution, and let it absorb for approximately 30 seconds (no more than 30 ).

6. Immediately inspect the color changes detected on the applicator and compare the color changes to the reference colors. Record negative results and positive color changes along with their locations on the report card.

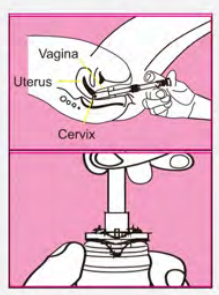

\section{Interpretation of the Result}

\section{After removing the applicator,} immediately inspect the color changes detected on the applicator and compare the color changes to the reference colors. Record negative results or positive results along with the locations of positive color changes on the report card.

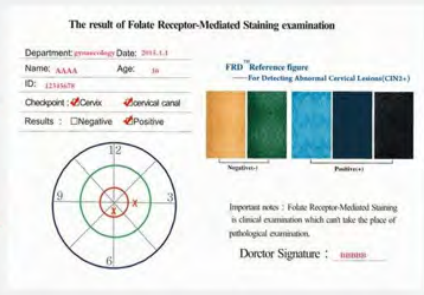

Figure 2 
$80 \%$, and $100 \%$ respectively. According to histopathological characteristics of CIN-2, the sensitivity of FRD was $79.2 \%$ and specificity $72.7 \%$ and accuracy $80.4 \%$ [15].

Compared with TCT and HPV testing, two usages of FRD have compatible sensitivity and high specificity to detect high grade cervical lesions and invasive carcinoma. FRD direct staining may be comfortable for routine cervical screening [16].

The advantages of FRD:

1. Cheap: Can be used in low resource areas.

2. Easy to perform.

3. Don't need a pathologist to interpret. Easy self-interpretation.

4. Immediate result (within 90 seconds). Hence avoids a second visit to collect the result.

5. Localizes the site of pathology, improving accuracy of biopsy.

6. Can detect changes in both squamous and columnar epithelium.

The disadvantages of FRD:

1. Results are influenced by bleeding during the procedure.

2. Not clear if the results can be affected by pregnancy.

\section{Conclusion}

FRD staining is a new, rapid, simple, accurate diagnostic method for screening of cervical precancerous lesions and early detection of cervical cancer. FRD is suitable for underdeveloped regions with large population and grassroot health institutions with insufficient medical technology.

Considering the health resources and women's preference, combination of FRD and Cytological evaluation may be chosen as a screening approach in developed countries.

\section{Bibliography}

1. De Martel C., et al. "Global burden of cancers attributed to infections in 2008:a review and synthetic analysis". The Lancet Oncology 13 (2012): 607-615.

2. Ferlay J., et al. "Estimates of world wide cancer in 2008. GLOBOCAN 2008”. International Journal of Cancer 127 (2010): 2893 to917.
3. Lu MH., et al. "A special epithelial staining agent: folic nacid receptor mediated diagnosis(FRD) effectively and conveniently screens patients with cervical cancer". International Journal of Clinical and Experimental Medicine 8 (2015): 7830-7836.

4. Yu Y., et al. "Folate receptor circulating tumor cells as a novel diagnostic biomarker in non small cell lung cancer". Translational Oncology 6 (2013): 967-702.

5. Coste J., et al. "Cross-sectional study of conventional cervical smear, monolayer cytology and human papilloma virus DNA testing for cervical cancer screening". British Medical Journal 326.7392 (2003): 733.

6. Passmore JA., et al. "Papnicalaou smear and cervical inflammatory cytokine response". Journal of Inflammation 4 (2007): 8.

7. Ronco G., et al. "Accuracy of liquid based versus conventional cytology: overall results of new technologies for cervical cancer screening randomized control trial". British Medical Journal 335.7609 (2007): 28.

8. Walboomers JM., et al. "HPV is a necessary cause of invasive cervical cancer worldwide". The Journal of Pathology 189.1 (1999): 12-19.

9. Cuschieri KS., et al. "Persistent high risk HPV infection associated with development of cervical neoplasia in a prespective population study". Journal of Clinical Pathology 58.9 (2005): 946-950.

10. Kulasinghan SC., et al. "Evaluation of HPV testing in primary screening for cervical abnormalities: comparison of sensitivity, specificity, and frequency of referral". The Journal of the American Medical Association 288.14 (2002): 1749-1757.

11. Cuzick J., et al. "Management of women who test positive for high risk type of HPV: the HART study". Lancet 362.9399 (2003): 1871-1876.

12. Sharples, Tiffany (2009-04-02) Times -HPV test screens best for cervical cancer. Times. Com Retrieved (2010).

13. Sankarnarayanan R., et al. "A critical method for screening of cervical neoplasia". International Journal of Gynaecology and Obstetrics 89 (2005): 54-512. 
14. International agency for research on cancer: a practical manual on visual screening for cervical neoplasia. Lyon, France IARC (2003).

15. Tao Gan., et al. The Practical Journal of Cancer 30 (2015).

16. Yuping Dai., et al. "Compare two different uses of FRD for detecting high grade cervical lesions and invasive cancer". Archives of Gynaecology and Obstetrics 299 (2019): 1399-1404.

\section{Assets from publication with us}

- Prompt Acknowledgement after receiving the article

- Thorough Double blinded peer review

- Rapid Publication

- Issue of Publication Certificate

- High visibility of your Published work

Website: www.actascientific.com/

Submit Article: www.actascientific.com/submission.php

Email us: editor@actascientific.com

Contact us: +919182824667 